\title{
Improved grey wolf algorithm for optimization problems
}

\author{
Hafiz Maaz Asgher', Yana Mazwin Mohmad Hassim², Rozaida Ghazali', Muhammad Aamir ${ }^{3}$ \\ ${ }^{1,2,3}$ Faculty of Computer Science and Information Technology, Universiti Tun Hussein Onn Malaysia (UTHM), Malaysia \\ ${ }^{3}$ School of Electronics, Computing and Mathematics, University of Derby, United Kingdom
}

\begin{tabular}{l}
\hline \hline Article Info \\
\hline Article history: \\
Received Jun 1, 2020 \\
Revised Aug 10, 2020 \\
Accepted Sep 25, 2020 \\
\hline
\end{tabular}

\section{Keywords:}

Exploitation

Exploration

Grey wolf optimization

Optimization

Whale optimization algorithm

\begin{abstract}
The grey wolf optimization (GWO) is a nature inspired and meta-heuristic algorithm, it has successfully solved many optimization problems and give better solution as compare to other algorithms. However, due to its poor exploration capability, it has imbalance relation between exploration and exploitation. Therefore, in this research work, the poor exploration part of GWO was improved through hybrid with whale optimization algorithm (WOA) exploration. The proposed grey wolf whale optimization algorithm (GWWOA) was evaluated on five unimodal and five multimodal benchmark functions. The results shows that GWWOA offered better exploration ability and able to solve the optimization problem and give better solution in search space. Additionally, GWWOA results were well balanced and gave the most optimal in search space as compare to the standard GWO and WOA algorithms.
\end{abstract}

This is an open access article under the CC BY-SA license.

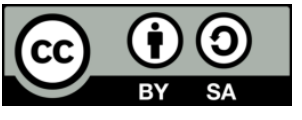

\section{Corresponding Author:}

Hafiz Maaz Asgher

Faculty of Computer Science and Information Technology

Universiti Tun Hussein Onn Malaysia (UTHM)

Parit Raja, 86400, Batu Pahat, Johor, Malaysia

Email: maazasghar96@gmail.com

\section{INTRODUCTION}

Optimization techniques play important role in engineering design, information science, economics management, operational research, and related areas [1], [2]. In computer science and mathematics, a problem is said to be an optimization problem; if, it has many viable solutions and the optimal solution is required to be found among all the feasible solutions by applying the least possible cost [3], [4]. Recently, metaheuristics algorithms have been used to solve many optimization problems. Some latest popularly used are particle swarm optimization (PSO) [5]-[7], artificial bee colony (ABC) [8], [9], cuckoo search algorithm (CSA) [10], [11], whale optimization algorithm (WOA) [12], firefly algorithm (FA) [13], ant colony optimization (ACO) [14] and bat algorithm (BA) [15].

This study focuses on the nature inspired grey wolf optimization (GWO) algorithm [12]. GWO is a swarm intelligent algorithm that follows the basic principles of leadership hierarchy and hunting behavior of grey wolves in nature. Due to its simplicity, GWO has been widely utilized to solve many practical optimization problems [16]-[20]. For a well-established solution search space generation, a fine balance is required between the exploration and exploitation processes for finding an optimized solution in a metaheuristic [21]. GWO algorithm is a metaheuristics algorithm that is unfortunately not able to ensure a fine balance between the exploration (finding new search space) and exploitation (finding solution in local search space) of the solution search space during the grey wolf position update stage [22]. This imbalanced relationship between the exploration and exploitation in GWO results in convergence towards degraded solution quality [23]. It also has poor exploration capability at small randomization which may lead to skip the most optimal solution and even the present solution [24]. In order to overcome this problem, whale 
optimization algorithm (WOA) is incorporated with GWO to improve the exploration capability, which in turn improves the global convergence results and ensures better quality solution.

Therefore, to overcome the problem of poor exploration in GWO, the initial population for the GWO is initialized using the searching prey mechanism of WOA. This ensures a well-balanced relationship between exploration and exploitation phase in the proposed grey wolf whale optimization algorithm (GWWO) algorithm. In the initial stage of the standard GWO it has the low convergence rate in the search space [23]. The position update equation performs well in exploitation and is not able to perform well during exploration [21]. In the grey wolf position change stage, the solution set generates the position based on the previous best position of alpha $(\alpha)$, beta $(\beta)$, and delta $(\delta)$ wolves [25]. At this stage, the ratio of exploitation and exploration randomly chose individuals from the population by a component called randomization factor that plays the most important role in changing the Grey wolf position it is used in turn to control the solution search of the optimization problem to be solved. The major role of this factor is to check the availability of the most optimal solution in vicinities of the current solution (exploitation). Also, it has to explore the solution search space to find new solution (exploration) is search of the most optimal solution [21].

\section{GREY WOLF OPTIMIZATION (GWO)}

GWO is a swarm intelligent algorithm that follows the basic principles of leadership hierarchy and hunting behavior of grey wolves in nature. Due to its simplicity, GWO has been widely utilized to solve many practical optimization problems [16]. GWO faces many challenging problems, it can easily get trapped in local optima because of imbalanced relationship between exploitation and exploration. Also, the positionupdate equation in GWO mostly relies on the information provided by the previous solutions to generate new candidate solutions which result in poor exploration activity [18]. Therefore, to overcome the problem of poor exploration in the GWO the exploration part of the whale optimization algorithm (WOA) is integrated in it [26]. The resultant grey wolf whale optimization algorithm (GWWOA) offers better exploration ability and can solve the optimization problems to find the most optimal solution in search space. The performance of the proposed algorithm is tested and evaluated on five benchmarked unimodal and five multimodal functions against GWO and WOA algorithms. The drawback of the standard GWO operation is its poor exploration capability at small randomization [24]. The poor exploration may lead to skip the most optimal solution and even the present solution [24]. The standard GWO is also poor in convergence rate and ultimately degrades the global solution quality [27]. In order to overcome this problem, WOA is incorporated with GWO that improve the exploration capability, which in turn improves the global convergence results and ensures better quality solution. Many researchers solved this problem using different ways to improve the global search of the GWO. WOA is rich in the exploration so its exploration part is used in GWO to improve the exploration. As compared to the other optimization techniques, WOA is easy to understand and implement. The flow diagram shows in Figure 1.

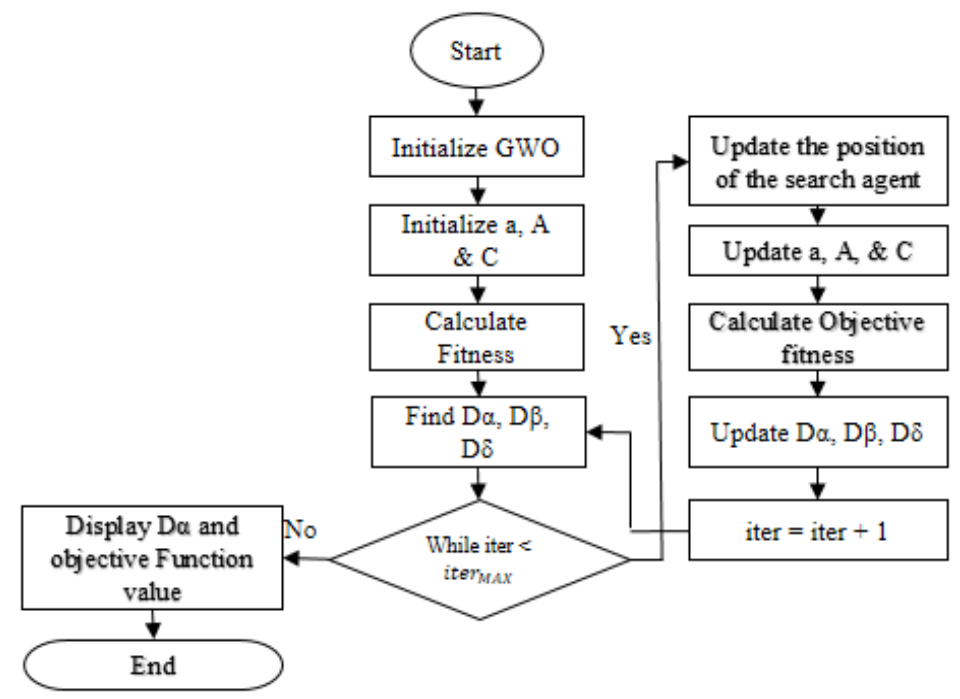

Figure 1. The GWO algorithm flow diagram 


\section{THE PROPOSED GWWOA ALGORITHM}

The aim of the study is to develop an integrated model of the GWO and WOA to balance the exploitation and exploration in the GWO. This balancing improves the quality of the GWO to solve the optimization problem. This section is dedicated to developing the model to resolve the problem in the standard model of GWO. The grey wolf whale optimization algorithm (GWWOA) is developed to solve different types of optimization problems. In this research, the target is to improve the ability of the exploration in Grey wolf optimization algorithm because the GWO algorithm performs well in exploitation but does not perform well in exploration. The objectives and scope of the research are mentioned in solving this problem. The proposed GWWOA algorithm performs well in both (exploitation and exploration). In GWWOA, the exploration process of WOA is integrated in the GWO to improve exploration performance in it because WOA perform well in exploration. The flow diagram of the proposed algorithm is shows as in Figure 2.

In this proposed algorithm, the WOA is an integral part of standard GWO, and used to improve the exploration part (equation) of GWO. The improvement in exploration part of GWO is due to its poor performance and imbalanced relationship with exploitation part of GWO. Further, standard GWO consisting of mainly three parameters which are "a", "A" and "c". In this research work, "a" was chosen as default value of 0.5 as described in WOA and varied to analyze the exploration part of GWO. Thus, the proposed GWWOA algorithm have change the parameter value from 1 to 0.5 for "a" as shown in vector "a" in pseudo code. On utilizing these variable values for parameter, the proposed GWWOA algorithm was used to improve exploration part of GWO. The simulation results revealed that the proposed GWWOA algorithm delivered balanced relationship between exploitation and exploration parts. Moreover, the parameter, " $A$ " used to express the decision for exploration and or exploitation parts of the GWO. And through the proposed GWWOA algorithm, if the value "A" obtained greater than 1, it will be exploration value. On the other hand, it will be exploitation value, when " $A$ " is less than 1 . Thus, the parameter "A" value was varied in this proposed GWWOA, as the exploration part of WOA performed better as compared to GWO.

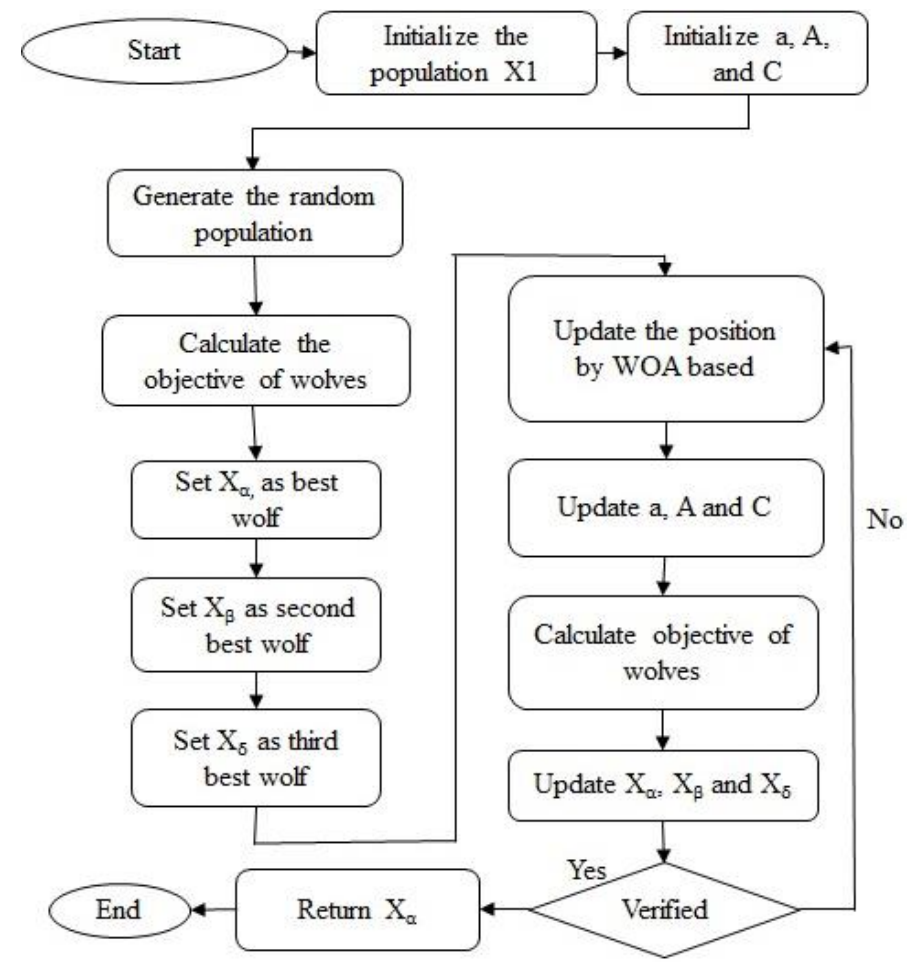

Figure 2. The proposed GWWOA algorithm flow diagram

\section{PERFORMANCE OF THE GWWOA ALGORITHM}

The different types of unimodal and multimodal benchmark functions are applied, and the performance is evaluated and compared to the standard GWO and WOA. The performance evaluation parameter of the proposed models for optimization function were best-case solutions, and standard deviation of solutions. All models and newly develop models are run 30 times for all the minimization and maximization functions and the optimal values obtained in each run are recorded. The best-case solution for 
minimization function is the minimum value among these 30 recorded values. The standard deviation shows the variations in these solutions. To conduct a fair comparison, both comparative and proposed algorithms are implemented using MATLAB 2017a software which are executed same hardware and software version running on core 17 CPU. Unimodal and multimodal functions are given in the Table 1 and Table 2, respectively.

Table 1. Unimodal function

\begin{tabular}{lccc}
\hline Function (s) & Dimension & Range & $f_{\min }$ \\
\hline$f_{1}(x)=\sum_{i=1}^{n} x_{i}^{2}$ & 30 & {$[-5.12,5.12]$} & 0 \\
$f_{2}(x)=-\exp \left(-0.5 \sum_{i=1}^{n} x_{i}^{2}\right)$ & 30 & {$[-1,1]$} & 0 \\
$f_{3}(x)=\sum_{i=1}^{n}\left|x_{i}\right|^{i+1}$ & 30 & {$[-10,10]$} & 0 \\
$f_{4}(x)=0.5+\frac{\sin ^{2}\left(x^{2}+y^{2}\right)-0.5}{\left[1+0.001\left(x^{2}+y^{2}\right)\right]^{2}}$ & 30 & {$[-100,100]$} & 0 \\
$f_{5}(x)=\sum_{i=1}^{n-1}\left[100\left(x_{i+1}-x_{i}^{2}\right)^{2}+\left(x_{i}-1\right)^{2}\right]$ & 30 & {$[-30,30]$} & 0 \\
\hline
\end{tabular}

Table 2. Multimodal function

\begin{tabular}{lccc}
\hline Function (s) & Dimension & Range & $f_{\min }$ \\
\hline$f_{6}(x)=418.9829 d-\sum_{i=1}^{n} x_{i} \sin \left(\sqrt{\left|x_{i}\right|}\right)$ & 30 & {$[-100,100]$} & 0 \\
$f_{7}(x)=10 n+\sum_{i=1}^{n}\left[x_{i}^{2}-10 \cos \left(2 \pi x_{i}\right)\right]$ & 30 & {$[-5.12,5.12]$} & 0 \\
$f_{8}(x)=-20 \exp \left(\sqrt[-0.2]{\frac{1}{n} \sum_{i=1}^{n} x_{i}^{2}}\right)-\exp \left(\frac{1}{n} \sum_{i=1}^{n} \cos \left(2 \pi x_{i}\right)\right)+20+e$ & 30 & {$[-32,32]$} & 0 \\
$f_{9}(x)=\frac{1}{4000} \sum_{i=1}^{n} x_{i}^{2}-\prod_{i=1}^{n} \cos \left(\frac{x_{i}}{\sqrt{i}}\right)+1$ & 30 & {$[-600,600]$} & 0 \\
$f_{10}(x)=\sum_{i=1}^{n}\left(\mathrm{e}^{-0.2} \sqrt{x_{i}^{2}+x_{i+1}^{2}}+3\left(\operatorname{coc}(2 \mathrm{xi})+\sin \left(2 x_{i+1}\right)\right)\right.$ & 30 & {$[-35,35]$} & 0 \\
\hline
\end{tabular}

\section{RESULTS AND DISCUSSION}

Based on the performance evaluation parameters, the developed model's simulation results are compared with the standard GWO and WOA. The results of the proposed model are better than standard GWO because GWO is good in attacking the prey and weak in searching the new prey. The proposed model solves this issue by balancing the exploitation and exploration.

\subsection{Unimodal Benchmark Functions}

First, the five unimodal benchmark functional dataset is applied to the proposed GWWOA to analysis the performance of the newly developed algorithm and compared the proposed model's results with the standard GWO and WOA algorithms. Five unimodal benchmark functions are applied to the proposed model. Table 3. Shows the results and its comparison. All the functions in Table 3. Have single local optimum in the trajectory of their search space. The maximum iterations for all the algorithms were set to 500 with a total of 30 trials on each function. Table 3. Shows the best-case value, and standard deviation value. From Table 3. The proposed GWWOA algorithm shows better results as compared to the other algorithm used for comparison. The GWWOA algorithm performed better on all five functions (i.e. $f 1$, $f 2$, $f 3, f 4$, and $f 5$ ). The comparison of the average convergence values obtained by the proposed GWWOA, GWO, In terms of convergence stability, the proposed GWWOA algorithm was the most stable during all the 30 run trials. Its stable performance can be seen through the standard deviation values given in the Table 3 .

Overall, the proposed GWWOA is found to be a highly stable algorithm during all the trial runs when compared with the standard GWO, and WOA and it gives better solutions on the unimodal benchmark functions in the search space.

\subsection{Multimodal Benchmark Functions}

The second step is the application of multimodal benchmark functions. The result of the proposed model is compared to the standard GWO and WOA algorithms. Five different multimodal benchmark functions are used to evaluate the performance of the developed model. The Table 4 shows the results and the 
comparison. During all the iterations the proposed model got the best values in the search space Table 4 shows the best-case solution, and standard deviation.

Table 4 shows the comparison of proposed algorithm GWWOA with the standard GWO and WOA. It shows the best results, and the standard deviation. The proposed algorithm GWWOA performed well during convergence to find out the most optimal solution in search space against the standard Grey Wolf Optimization and Standard Whale Optimization algorithms. The proposed GWWOA algorithm converged to average global optimal values of 3.01E-02, 1.02E-09, 6.19E-16, 1.45E-01, and 3.01E-01 for functions $f 6, f 7$, $f 8, f 9$, and $f 10$, respectively. The results show the stability of the proposed GWWOA algorithm through its standard deviation results on multimodal benchmark functions $f 6$ to $f 10$.

Table 3. Performance evaluation for unimodal

\begin{tabular}{cccc}
\hline Function $(f)$ & Algorithm & $\begin{array}{c}\text { Best Case } \\
\text { Value }\end{array}$ & $\begin{array}{c}\text { Standard } \\
\text { Deviation Value }\end{array}$ \\
\hline$f 1$ & GWO & $1.05 \mathrm{E}-17$ & $6.24 \mathrm{E}-09$ \\
& WOA & $2.26 \mathrm{E}-27$ & $2.09 \mathrm{E}-24$ \\
$f 2$ & GWWOA & $3.68 \mathrm{E}-28$ & $9.41 \mathrm{E}-24$ \\
& GWO & $5.20 \mathrm{E}-19$ & $3.20 \mathrm{E}-18$ \\
& WOA & $1.33 \mathrm{E}-27$ & $3.71 \mathrm{E}-22$ \\
$f 3$ & GWWOA & $5.90 \mathrm{E}-33$ & $2.03 \mathrm{E}-24$ \\
& GWO & $4.08 \mathrm{E}-04$ & $2.83 \mathrm{E}-02$ \\
& WOA & $5.36 \mathrm{E}+01$ & $3.82 \mathrm{E}+02$ \\
& GWWOA & $3.81 \mathrm{E}-06$ & $2.33 \mathrm{E}-03$ \\
& GWO & $1.91 \mathrm{E}-04$ & $1.32 \mathrm{E}-03$ \\
& WOA & $1.74 \mathrm{E}-04$ & $1.40 \mathrm{E}-03$ \\
& GWWOA & $1.41 \mathrm{E}-04$ & $7.71 \mathrm{E}-04$ \\
& GWO & $2.61 \mathrm{E}-02$ & $7.88 \mathrm{E}-02$ \\
& WOA & $4.81 \mathrm{E}-02$ & $2.40 \mathrm{E}-02$ \\
& GWWOA & $4.9 \mathrm{E}-02$ & $2.46 \mathrm{E}-02$ \\
\hline T* The highlighted cells contain the proposed algorithm's results
\end{tabular}

Table 4. Performance evaluation for multimodal

\begin{tabular}{cccc}
\hline Function $(f)$ & Algorithm & $\begin{array}{c}\text { Best Case } \\
\text { Solution }\end{array}$ & $\begin{array}{c}\text { Standard } \\
\text { Deviation }\end{array}$ \\
\hline$f 6$ & GWO & $4.24 \mathrm{E}-02$ & $1.44 \mathrm{E}-01$ \\
& WOA & $4.23 \mathrm{E}-02$ & $1.05 \mathrm{E}-02$ \\
& GWWOA & $4.35 \mathrm{E}-02$ & $6.71 \mathrm{E}-03$ \\
$f 7$ & GWO & $9.96 \mathrm{E}-01$ & $4.70 \mathrm{E}-01$ \\
& WOA & $8.00 \mathrm{E}-09$ & $6.12 \mathrm{E}-09$ \\
& GWWOA & $7.80 \mathrm{E}-09$ & $3.49 \mathrm{E}-09$ \\
$f 8$ & GWO & $4.28 \mathrm{E}-16$ & $2.48 \mathrm{E}-14$ \\
& WOA & $6.09 \mathrm{E}-15$ & $6.40 \mathrm{E}-16$ \\
\multirow{4}{*}{$f 9$} & GWWOA & $5.19 \mathrm{E}-16$ & $9.80 \mathrm{E}-17$ \\
& GWO & $9.38 \mathrm{E}-01$ & $2.97 \mathrm{E}-01$ \\
& WOA & $9.78 \mathrm{E}-01$ & $2.88 \mathrm{E}-01$ \\
\multirow{2}{*}{$f 10$} & GWWOA & $5.84 \mathrm{E}-01$ & $3.23 \mathrm{E}-01$ \\
& GWO & $3.48 \mathrm{E}-02$ & $1.36 \mathrm{E}-01$ \\
& WOA & $4.69 \mathrm{E}-03$ & $1.73 \mathrm{E}-01$ \\
& GWWOA & $4.89 \mathrm{E}-03$ & $1.72 \mathrm{E}-01$ \\
\hline ** The highlighted cells contain the proposed algorithm's results
\end{tabular}

\section{CONCLUSION}

GWO is an innovative optimization algorithm and is inspired from hunting and hierarchy behavior of grey wolves' group. GWO is mainly composed of three main positions including alpha, beta and omega, which followed each other to solve optimization problems. In this research work, the GWWOA performance was compared with standard GWO and WOA through total ten benchmark functions. The central idea of this research work was to solve the problems related to exploration in GWO through the help from exploration part of the WOA. The GWWOA results revealed better accuracy and were in good agreement with standard GWO and WOA. Further, the GWWOA algorithm solved optimization problem and found optimized solution in search space. Additionally, the GWWOA algorithm showed better convergence solution as compared to standard GWO and WOA and had well balance in exploitation and exploration. Moreover, the GWWOA algorithm improved the solution time as compared to the consumed time of GWO and WOA algorithms. Finally, as based on the obtained results, the GWWOA is suitable algorithm to solve optimization issues. 


\section{ACKNOWLEDGEMENTS}

This research was supported by ministry of higher education (MOHE) through fundamental research grant scheme (FRGS/1/2020/ICT02/UTHM/02/1) Vot K270.

\section{REFERENCES}

[1] B. Mahdad and K. Srairi, "Blackout risk prevention in a smart grid based flexible optimal strategy using Grey Wolf-pattern search algorithms," Energy Convers. Manag., vol. 98, pp. 411-429, 2015, doi: 10.1016/j.enconman.2015.04.005.

[2] J. A. Koupaei, S. M. M. Hosseini, and F. M. M. Ghaini, "A new optimization algorithm based on chaotic maps and golden section search method," Eng. Appl. Artif. Intell., vol. 50, pp. 201-214, 2016, doi: 10.1016/j.engappai.2016.01.034.

[3] F. Wahid et al., "An Enhanced Firefly Algorithm Using Pattern Search for Solving Optimization Problems," IEEE Access, vol. 8, pp. 148264-148288, 2020, doi: 10.1109/ACCESS.2020.3015206.

[4] P. Olivella-Rosell et al., "Optimization problem for meeting distribution system operator requests in local flexibility markets with distributed energy resources," Appl. Energy, vol. 210, pp. 881-895, Aug. 2018, doi: 10.1016/j.apenergy.2017.08.136 .

[5] T. Kezong, Z. Li, L. Luo, and B. Liu, "Multi-Strategy Adaptive Particle Swarm Optimization for Numerical Optimization," Engineering Applications of Artificial Intelligence, vol. 37, pp. 9-19. 2015, doi: 10.1016/j.engappai.2014.08.002.

[6] R. Jensi, and G. W. Jiji, "An enhanced particle swarm optimization with levy flight for global optimization," Applied Soft Computing, vol. 43, pp 248-261, 2016, doi: 10.1016/j.asoc.2016.02.018 .

[7] Z. Zhan, J. Zhang, Y. Li and Y. Shi, "Orthogonal Learning Particle Swarm Optimization," in IEEE Transactions on Evolutionary Computation, vol. 15, no. 6, pp. 832-847, Dec. 2011, doi: 10.1109/TEVC.2010.2052054.

[8] F. Zhong, L. Hui, and S. Zhong, "An improved artificial bee colony algorithm with modified-neighborhood-based update operator and independent-inheriting-search strategy for global optimization," Engineering Applications of Artificial Intelligence, vol. 58, pp 134-56, 2017, doi: 10.1016/j.engappai.2016.11.005.

[9] A. Yurtkuran, and E. Emel, "An Adaptive Artificial Bee Colony Algorithm for Global Optimization," Applied Mathematics and Computation, vol. 271, pp. 1004-1023, 2015, doi: 10.1016/j.amc.2015.09.064.

[10] H. Rakhshani, and A. Rahati, "Snap-Drift Cuckoo Search: A Novel Cuckoo Search Optimization Algorithm," Applied Soft Computing Journal, vol. 52, pp. 771-94, 2017, doi: 10.1016/j.asoc.2016.09.048.

[11] U. Mlakar, I. Fister Jr, and I. Fister, "Hybrid self-adaptive cuckoo search for global optimization" Swarm and Evolutionary Computation. vol 29, pp 47-72, 2016.

[12] S. Mirjalili, S. Mohammad, and A. Lewis, "Grey Wolf Optimizer," Adv. Eng. Softw., vol. 69, pp. 46-61, 2014, doi: 10.1016/j.advengsoft.2013.12.007.

[13] A.H. Gandomi, X.S. Yang, S Talatahari, and A H Alavi, "Firefly algorithm with chaos," Communications in Nonlinear Science and Numerical Simulation, vol. 18, no. 1, pp. 89-98, 2012, doi: 10.1016/j.cnsns.2012.06.009.

[14] Z. Chen, , S. Zhou, and J. Luo, "A robust ant colony optimization for continuous functions," Expert Systems with Applications, vol. 81, pp. 309-320, 2017, doi: 10.1016/j.eswa.2017.03.036.

[15] A. Chakri, R. Khelif, M. Benouaret, \& X.S. Yang, "New directional bat algorithm for continuous optimization problems," Expert Systems with Applications, vol. 69, pp. 159-175, 2017, doi: 10.1016/j.eswa.2016.10.050.

[16] İ. Gölcük and F. B. Ozsoydan, "Evolutionary and adaptive inheritance enhanced Grey Wolf Optimization algorithm for binary domains," Knowledge-Based Systems, Vol. 194, 2020, doi: 10.1016/j.knosys.2020.105586.

[17] N. Jayakumar, S Subramanian, S Ganesan, and E B Elanchezhian, "Grey Wolf Optimization for Combined Heat and Power Dispatch with Cogeneration Systems," Int. Journal of Electrical Power and Energy Systems, vol. 74, pp. 252-64, 2016, doi: 10.1016/j.ijepes.2015.07.031.

[18] M. Fahad, F. Aadil, Z. Rehman, S. Khan, P A Shah, M. Khan , J. Lloret, H. Wang, J W Lee, I Mehmood, "Grey Wolf Optimization Based Clustering Algorithm for Vehicular Ad-Hoc Networks," Computers and Electrical Engineering, vol. 70, pp. 853-70, 2018, doi: 0.1016/j.compeleceng.2018.01.002 .

[19] X. Song, L Tang, S Zhao, X Zhang, L Li, J Huang, and W Cai, "Grey Wolf Optimizer for Parameter Estimation in Surface Waves," Soil Dynamics and Earthquake Engineering, vol. 75, pp. 147-57, 2015, doi: 10.1016/j.soildyn.2015.04.004.

[20] A. A. Heidari and P. Pahlavani, "An efficient modified grey wolf optimizer with Lévy flight for optimization tasks,” Appl. Soft Comput. J., vol. 60, pp. 115-134, 2017, doi: 10.1016/j.asoc.2017.06.044.

[21] W. Long, J. Jiao, X. Liang, and M. Tang, "An exploration-enhanced grey wolf optimizer to solve high-dimensional numerical optimization," Eng. Appl. Artif. Intell., vol. 68, pp. 63-80, Apr. 2018, doi: 10.1016/j.engappai.2017.10.024.

[22] Z. Teng, J Lv, and L Guo, “An Improved Hybrid Grey Wolf Optimization Algorithm,” Soft Computing, vol. 23, no. 15, pp. 6617-31, 2019, doi: 10.1007/s00500-018-3310-y.

[23] S. Dhargupta, M. Ghosh, S. Mirjalili, and R. Sarkar, "Selective Opposition based Grey Wolf Optimization," Expert Syst. Appl., vol. 151, pp. 113389, 2020, doi: 10.1016/j.eswa.2020.113389.

[24] M.A.M. Majeed, and S. R. Patri. "An Enhanced Grey Wolf Optimization Algorithm with Improved Exploration Ability for Analog Circuit Design Automation," Turkish Journal of Electrical Engineering and Computer Sciences, vol. 26, no. 5, pp. 2605-2617, 2018, doi: 10.3906/elk-1802-110 . 
[25] S. Mirjalili and A. Lewis, "The Whale Optimization Algorithm," Advances in engineering software, vol. 95, pp. 51-67, 2016, doi: 10.1016/j.advengsoft.2016.01.008.

\section{BIOGRAPHIES OF AUTHORS}
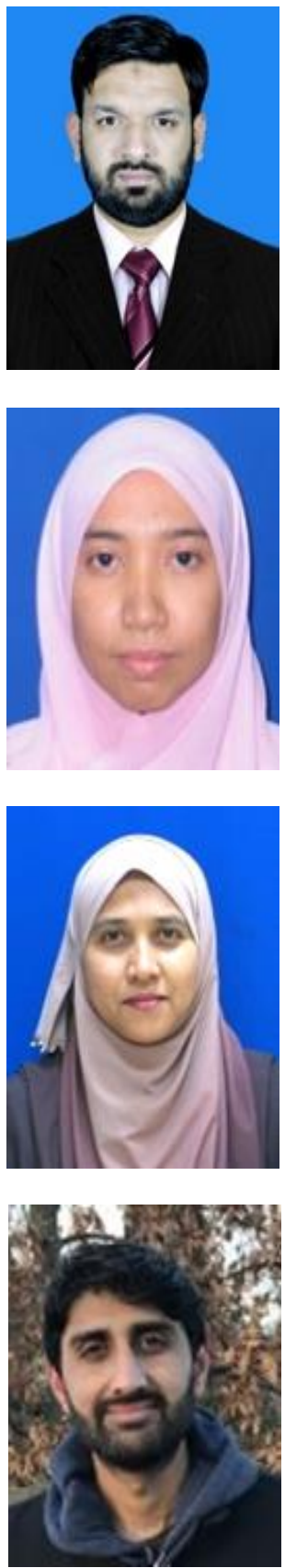

Hafiz Maaz Asgher belongs to Rahim Yar Khan, Punjab, Pakistan. He holds a Bachelor degree in Computer Science and Information Technology from The Islamia University of Bahawalpur (IUB) in 2015. Currently he is pursuing Master's degree from Faculty of Computer Science and Information Technology, Universiti Tun Hussein Onn Malaysia (UTHM). His research interests includes optimization, machine learning and artificial intelligence

Yana Mazwin Mohmad Hassim is a senior lecturer at the Faculty of Computer Science and Information Technology, Universiti Tun Hussein Onn Malaysia (UTHM). She graduated with a PhD degree from Universiti Tun Hussein Onn Malaysia (UTHM) in 2016. Earlier, in 2006 she completed her Master's degree in Computer Science from Universiti of Malaya (UM). She received her Bachelor of Information Technology (Hons) degree majoring in Industrial Computing from Universiti Kebangsaan Malaysia (UKM) in 2001. In 2003, Yana Mazwin joined the academic staff in UTHM. Her research area includes neural networks, swarm intelligence, optimization and classification.

Rozaida Ghazali is currently a Professor at the Faculty of Computer Science and Information Technology, Universiti Tun Hussein Onn Malaysia (UTHM). She graduated with a Ph.D. degree in Higher Order Neural Networks from the School of Computing and Mathematical Sciences at Liverpool John Moores University, United Kingdom in 2007. Earlier, in 2003 she completed her M.Sc. degree in Computer Science from Universiti Teknologi Malaysia (UTM). She received her B.Sc. (Hons) degree in Computer Science from Universiti Sains Malaysia (USM) in 1997. In 2001, Rozaida joined the academic staff in UTHM. Her research area includes neural networks, swarm intelligence, optimization, data mining, and time series prediction.

Muhammad Aamir has recently received his $\mathrm{PhD}$ in Information Technology from Universiti Tun Hussein Onn Malaysia. He did his Master's degree in Computer Science from City University of Science and Information Technology Pakistan. He had worked for two years in Xululabs LLC as data scientist. Currently he is working on research related to big data processing and data analysis. His fields of Interest are Data Science, Deep Learning, and Computer Programming. 